\title{
El tratado de documentación de Paul Otlet: una exposición metacientifica
}

\author{
Leomar José Montilla Peña \\ Universidad Politécnica Territorial Andrés Eloy Blanco - Venezuela
}

ANÁLISIS / ANALYSIS

\begin{abstract}
Resumen
Se presenta un análisis descriptivo del Tratado de Documentación de Paul Otlet, señalando algunos aspectos significativos. Para ello, se delimita, en primer lugar, el Tratado de documentación como expositor discursivo de esa moderna ciencia propuesta por Otlet. A continuación se examina de forma analítica su objeto de estudio y por último se establece la noción de documentación presentada.
\end{abstract}

Palabras clave

Tratado de Documentación ; Paul Otlet ; Documento ; Documentación

\section{The treaty of documentation of Paul Otlet: a metascientific exposure}

\section{Abstract}

We present a descriptive analysis of the Treaty Documentation Paul Otlet, noting some significant aspects. This involves defining, first, the Treaty as an exhibitor documentation that modern science discourse proposed by Otlet. Then analytically examines its subject matter and finally establishes the notion of documentation submitted.

Keywords

Treaty of Documentation ; Paul Otlet ; Document ; Documentation

\section{Introducción}

Esta breve exposición tiene por objetivo discurrir sobre uno de los hechos más significativo de las corrientes científicas que estudian la información registrada y que tuvo su origen durante la década de los años treinta del siglo XX. El artículo se refiere a la ciencia moderna propuesta por Paul Otlet a través de su Tratado de Documentación donde expone una metaciencia que esta más allá de las prácticas científicas tradicionales de la bibliotecología, la archivología y la museología de acuerdo a su contexto, donde Otlet logra articular la ciencia, la tecnología y la sociedad originando así una ciencia moderna de carácter universal.

Es evidente que poco se ha escrito sobre su existencia en el contexto Latinoamericano, específicamente en el idioma español. Por esta razón, esta investigación busca brindar una noción sobre la Ciencia de la Documentación propuesta por Otlet en su Tratado para dar a conocer su contenido y de este manera llamar a la reflexión y discusión 
académica entre estudiantes, docentes y profesionales de la información sobre este hecho, a partir de un escrito disponible y fácilmente útil.

Lo anterior explica el interés de presentar este artículo haciendo énfasis en la importancia del Tratado de Documentación como referente bibliográfico para la compresión de los procesos interdisciplinarios y transdisciplinarios que ocurren en la actualidad en estas ciencias que estudian la información registrada y de esta forma ayudar al mejoramiento de estas.

En primer lugar, ofrecemos una breve síntesis sobre El Tratado de Documentación y su estructura como expositor discursivo de esa moderna ciencia. En segundo lugar, referimos el objeto de estudio de la ciencia propuesta por Otlet, por ultimo presentamos la noción de documentación planteada en el Tratado de Documentación.

\section{El Tratado de Documentación y su estructura}

El inicio del siglo XX sorprendió a la Bibliotecología, la Archivología y la Museología con una revolución tecnológica que marcaría para siempre sus desarrollos. La aparición del telégrafo, el teléfono, la radio, el cine, las máquinas de escribir y la fotografía, cambiarían para siempre las formas de registrar información. En consecuencia, estas ciencias se vieron obligadas a lidiar con nuevos tipos de documentos, gráficos, sonoros, de imágenes fijas y en movimiento, muy diferentes de las formas tradicionales fijas de registro de información sobre papel con las que tradicionalmente habían lidiado estas ciencias. Es precisamente en este complejo escenario, por demás fuertemente influido por los enfoques positivistas inspirados en la idea filosófica de la verdad propia de la modernidad, en la que se inserta la monumental obra científica de Paul Marie Ghislain Otlet (1868-1944). Al punto de que se le considera "...un visionario cuyas ideas estaban por lo menos cincuenta años por delante de su tiempo." (Rayward, 1975, p.3, traducido por el autor) Otlet, como se le ha conocido después en los escenarios académicos, se dedicó especialmente a organizar el conocimiento registrado y las instituciones documentales de su país, generando una gran cantidad de documentos donde dejó plasmadas novedosas teorías y metodologías para alcanzar tal fin. En 1934 publicó, en Bruselas, su obra más destacada y la que ha traspasado con creces la frontera del tiempo: El Tratado de Documentación. El libro sobre el libro. Teoría y práctica.

Siendo esta obra uno de sus mayores logros intelectuales, como género literario y como órbita didáctica, de cara a los avances ocurridos a finales del siglo XIX y principios del siglo XX en el campo de la bibliotecología, la archivología y la museología, a lo largo del Tratado Otlet ejerce un novedoso discurso enciclopédico y psicolingüístico en el que expone sus puntos de vistas sobre el documento y la documentación. Por tanto, en las propias palabras de Emile Verhaeren el tratado es "... una exposición general de las nociones relativas al libro y al documento, al empleo razonado de los elementos que constituyen la documentación" (Otlet, 2004, p.3).

Sin duda, Otlet buscó constituir, difundir y consolidar a través de su Tratado de Documentación un saber científico que fuera más allá de los presupuestos tradicionales de la Bibliotecología. Para ello utilizó diferentes términos para denominarla, entre ellos: bibliología, documentación o ciencias del libro y la documentación, como expresiones representativas de su moderna teoría de carácter universal. Universalidad explicada por López (1995) ampliamente cuando afirma "... universalidad en el tratamiento del concepto de la documentación, universalidad en el concepto de los documentos, universalidad en todo lo relativo a la organización institucional e internacional de la documentación" (p.77).

Ante el uso y aplicación de los términos que de forma indistinta hace Otlet (2004) para describir la ciencia de la documentación en el Tratado, es importante señalar que su enfoque, es el documento, describiendo a este como "el medio de transmitir datos informativos para el conocimiento de los interesados, que alejados en el tiempo y en el espacio, o cuyo espíritu discursivo necesita que se le muestren los vínculos inteligibles de las cosas" (p. 25). En este sentido, adopta según Rayward "...una creencia generalizada modernista en la racionalidad, la planificación, la normalización, la mecanización" (Rayward, 2008, p. 14, citado en Espley, 2011, traducido por el autor). 
Su inspirada obra deja ver su complejidad, su diversidad y sistematización para tener una comprensión completa de la documentación ante el problema que subsiste con el libro, el documento y labor de documentar a la sociedad durante ese periodo y los próximos, ilustrando de una forma casi absoluta todos los aspectos del libro y de los documentos en cualquier soporte y representación "... analizando los lugares de custodia, las formas de ordenamiento y codificación, así como la gestión de los documentos” (Cabrales y Linares, 2005, p. 88).

Lo que permite tener una comprensión dogmática desde tres puntos de vistas diferentes; como ciencia ya que estudia todos los aspectos del documento y como pueden ser estudiados, como técnica porque comprende las reglas y las instrucciones para la producción, circulación, conservación y utilización de los documentos y como organización puesto que permite la distribución de las fuerzas individuales y del trabajo colectivo que contribuyan al desarrollo de otras ciencias. (Otlet, 2004, p.11).

Al mismo tiempo, Otlet considera los avances tecnológicos y los inventos que surgieron durante esa época y los próximos, como medios para transmitir, comunicar y difundir a la sociedad, sobre los documentos existentes en las instituciones documentales. Igualmente los progresos en materia administrativa para fortalecer la ciencia y las técnicas generales que declara en su obra.

Por ello, se puede apreciar El Tratado con "una visión amplia e integradora de bibliografía y documentación, a través de normas y la búsqueda de la eficiencia, la gestión de recursos de información en cualquier contexto aplicable..." (Bucklan, 2008, p.8, traducido por el autor).

Desde esta perspectiva, El Tratado de Documentación queda constituido en cinco partes, precedida por la presentación y los fundamentos en el cual expresa en un ámbito más formal, general y ecuménico que lo visto anteriormente.

En la primera parte, Otlet expone los principios sustanciales de la Documentación o Bibliología adecuadas al contexto y los tiempos venideros como punto de partida para su teoría general de la documentación, donde define sus características, divisiones, problemas, métodos de investigación, interdisciplinariedad, historia y evolución.

En la segunda parte, Otlet realiza una minuciosa explicación sobre el libro y el documento para poner en mano del lector una mayor compresión universal, es decir, como una unidad individual específica y como un conjunto en su totalidad. Para ello recurre a definiciones, características, tipos de soportes, aspectos tecnológicos, funciones, operaciones, actividades y las instituciones que han sido las responsables de su organización y distribución (archivos, bibliotecas, centros de documentación y museos).

En la tercera parte, Otlet realiza un enfoque desde la bibliología compara advirtiendo sobre los libros como unidades individuales o en conjuntos, determinando su fondo y forma a través de un recorrido histórico, técnico y social.

Mientras que en la cuarta parte del Tratado, Otlet enuncia los principios y métodos para la organización racional del libro y el documento, formula las normas concretas de los métodos que describe en la primera parte del Tratado para poner en práctica la documentación en las diferentes instituciones documentales. Además, propone una red universal de información y documentación donde está a su vez sirva de enseñanza entre todas las instituciones que la conformen, indistintamente de su ubicación geográfica.

Finaliza el Tratado exponiendo las leyes generales de la bibliología y su relación con otras ciencias, además de insertar la ciencia de la documentación dentro de las ciencias sociales, describiendo también algunas visiones futuristas sobre la evolución del libro y el documento.

Es importante señalar que según Rayward "...Esta estructura cubre nuevas formas de mirar y hablar sobre los aspectos del mundo del conocimiento, los libros y las bibliotecas, y la infraestructura social de la que forma parte. Esta compleja interrelación de sistemas y racionalización establecieron lo que podemos llamar, después de Foucault, una nueva "formación discursiva" (Rayward, 1997, p. 289, traducido por el autor).

En resumen, Otlet fijó un equilibrio entre las ciencias técnicas y las ciencias sociales, fundamentando una metaciencia a la cual designó con el término documentación, y cuyo enfoque se enmarca desde una convicción y representación más amplia que la del libro. Ofreció una apertura racional abierta, no reduccionista, necesaria para la comprensión del documento como cosa informativa, comunicativa y preservacionista desde tres dimensiones: lo que muestra, lo que promueve y lo que prueba un individuo o unos individuos a una sociedad determinada, con el 
propósito de que estos puedan tener conocimiento en determinadas área del saber humano y puedan pronunciar su aceptación o refutación, indistintamente de su soporte, medio de registro, tiempo y espacio.

Toda esta determinación metacientifica se aprecia en el Tratado por el orden lógico de su discurso percibiéndose teorías fácticas y relevancia empírica, donde Otlet establece metodología y técnicas para la documentación asentada en los fundamentos epistemológicos, ontológicos y éticos de la investigación científica, concibiendo de esta toda una doctrina, sin embargo la mayor determinación de la metaciencia propuesta por Otlet -investigador- se parecía en la estrecha relación con el documento -lo observado- concibiendo una moderna ciencia sobre la base del constructo social donde esta debe estar en constante perfeccionamiento de acuerdo a los cambios que se generen en el contexto social.

\title{
3. El documento como objeto de estudio establecido por Otlet en el Tratado de Documentación
}

El Tratado de Documentación tiene un común denominador, el estudio del documento. Para este objeto de estudio Otlet aporta lo que en su visión define como cosa informativa, sobre la cual construye todo una concepción epistemológica, teórica, metodológica y técnica que difería del concepto de documento que había sido hegemónico en la Bibliotecología y la Archivología.

Quizás la mejor exponente sobre esta hegemonía es la profesora Blanca Rodríguez Bravo en su obra El documento. Entre la tradición y la renovación, quien nos da una representación más explícita y sintética al describirnos que:

\begin{abstract}
La dicotomía estaba clara: documento (archivo)/libro (biblioteca). Cada uno de estos centros tenían sus funciones, sus documentos particulares y sus formas de tratamiento [...] el documento de archivo y el de biblioteca no se diferencian en su forma sino en su origen. (Rodríguez, 2002, p. 14, citado en Malheiro, 2006, traducido por el autor).
\end{abstract}

Esta misma autora amplía más aún este punto sobre la supremacía del documento de este binomio científico en su artículo Revisión de las clasificaciones documentales basadas en el soporte, donde manifiesta que:

La principal oposición se establecía entre los documentos en papel, que dominaban el mercado de la edición, a los que se denominaba documentos impresos, bibliográficos o librarios normalmente, y el resto de los documentos, que eran producto de los avances técnicos producidos en el siglo $\mathrm{XX}$, y que se agrupaban, a efectos prácticos, bajo denominaciones en absoluto acertadas: documentos especiales, no impresos o no librarios, traduciendo de las expresiones inglesas «non print materials» 0 «non-book materials» (Rodríguez, 2002, p. 83).

Otro punto tratado sobre el posicionamiento cerrado del objeto de estudio de la bibliotecología y la archivología, es explicado por Profesor Armando Malheiro (2006) al afirmar que desde el "Renacimiento hasta la llustración opera una división entre el documento y el monumento, convirtiéndose en uno de los rehenes de la escuela histórica positivista a finales del siglo XIX y principios del siglo XX" (p. 49, traducido por el autor).

Estudiando estas particulares y otros elementos que predominan en la bibliotecología y la archivología, Otlet trata de corregir lo que él consideraba efectos indeseados en la prestación de los servicios para la sociedad y que era causada por la limitación del objeto de estudio de estas ciencias, sus técnicas de investigación y sus procesos informativos y comunicativos. Por lo que Otlet buscó reproducir en el Tratado de Documentación una exposición objetiva, sistemática y crítica del documento, reivindicando a éste desde una dimensión más amplia como medio informativo y comunicativo indistintamente de sus soportes y medios de representación. 
Basado en toda complejidad del documento por sus caracteres internos y externos, su importancia informativa para la sociedad y el conocimiento que los individuos desean transmitir por medio de el, Otlet establece métodos para estudiar la realidad del fenómeno documental y conocer sus funciones y fines al servicio de la sociedad.

En este sentido, Otlet define al documento como "Expresiones escritas de las ideas, instrumentos de su fijación, de su conservación, de su circulación, son los intermediarios obligados de todas las relaciones entre los hombres" (Otlet, 2004, p. 3)

Desde su punto de vista y analizando el problema detectado en cuanto al libro y el documento administrativo como únicos portadores de información y conocimiento, Otlet plantea dos premisas esenciales que trasciende la definición de documento:

\begin{abstract}
Hay ocasión de constituir la terminología a partir de la palabra "documento" más general que libro o biblión; este cambio de radical está justificado: $1^{\circ}$ por los motivos que han hecho admitir la palabra documento, documentación. $2^{\circ}$ por el retraso de las prácticas del mundo del libro, que, no habiendo evolucionado con suficiente rapidez, ha dejado crearse toda una nomenclatura, en parte para los objetos y nociones de los que había desinteresado al principio. (Ibídem).
\end{abstract}

Al pronunciar estas premisas, Otlet logró contrastar sus ideas con la realidad del fenómeno libro/documento que la Bibliotecología y la Archivología habían mantenido intacto desde el siglo XIX hasta el momento en que escribe su obra, el libro, visto como único objeto de estudio por la biblioteconomía y defendido como la supremacía del conocimiento humano. Esto condujo a Otlet considerar el término documento como el único objeto a construir en el nuevo saber científico que proponía y lo veía de una forma más general y absoluto.

Como resultado, Otlet subraya que "El documento elemental corresponde al pensamiento discursivo. Sirve de apoyo a este pensamiento permitiéndoles un desarrollo explícito cada vez más extendido y abundante. El documento es el grado superior que se entrevé correspondería al pensamiento intuitivo" (Ob. Cit. p. 11).

Desde esta perspectiva, el documento es un vocablo que no tiene denotación precisa alguna, y que tiene múltiples connotaciones según las múltiples corrientes del pensamiento dentro de cada una de esos dominios que le dan diverso sentido a la palabra documento, por ello, Otlet sugiere pensar en un sentido más amplio del concepto tradicional de documento y asociarlo a otras dimensiones de la satisfacción humana para tener una idea más completa y trascendental sobre él.

\footnotetext{
"Así, para Otlet el documento se encuentra en el centro de un complejo proceso de la comunicación, de la acumulación y transmisión del conocimiento, de la creación y evolución de las instituciones" (Rayward, 1991, p. 135, traducido por el autor)
}

Lo que evidencia la necesidad del estudio y el pensamiento del documento desde su utilidad, su medición y organización, sobre la base de esta idea Otlet considera que "Hay que hacer una distinción primera y fundamental entre la cosa y su ciencia, aquí entre el mismo libro-documento y la ciencia libro-documento" (Otlet, 2004, p.32). Rayward nos permite entender mejor esta idea genial de Otlet: "La ciencia es, por supuesto, el conjunto de todos los hechos observados y todas las hipótesis probables sugeridas para explicar tales hechos estudiados y su relación con las leyes" (Rayward, 1975, p.35, traducida por el autor).

Como puede verse, la hipótesis y su verificación serán los instrumentos de trabajo en El Tratado de Documentación. Representando una guía para la investigación y de técnicas que se encuentran relacionadas con las diversas formas del conocimiento, por ello la definición del documento y del fenómeno documentación comparado con el libro y la bibliotecología, forman una de las partes más importantes para el dominio del objeto y ese nuevo accionar científico.

Es en este sentido, que Otlet unificó el término de libro y documento para declarar un solo objeto de estudio, ya que consideraba que estos términos se habían confundido históricamente, y sin embargo, existía entre ellos una relación necesaria. El concepto de Otlet sobre documento como objeto hace referencia a lo que él busca construir a través de la disciplina científica como objeto del conocimiento y como ciencia moderna. 
Es por ello que "Para constituir un método -el de la descripción de los libros y de los estudios de los conjuntos de libros- Ilega pronto ampliarse el concepto que hace del mismo libro hasta sustituirlo por el concepto de documento". Esa nueva forma de pensamiento, aportada por Otlet en el Tratado, rebasó metodológicamente la concepción de percepción del libro en cuanto a su carácter informativo "ya no es ni la de la biblioteconomía ni la de la bibliografía, es la del conjunto del libro y del documento, la documentación"(Otlet, 2004, p.38).

A este respecto, López Yepes apunta que para Otlet "el enfoque científico del documento recoge los principios antropológicos y culturales [...] presta el objeto de estudio a la Documentación por cuanto es constitutivo esencial del proceso de la documentación, portador y transmisor de mensajes registrados y recuperables, y naturaleza sujeta a proceso de transformación" (López, 1997, p.15).

El unificar este binomio y buscar una sinonimia entre ambos es en realidad un aporte dialectico exclusivo de Otlet, lo que supone usos comunes y presupone ideas comunes, lo que conduce naturalmente a considerar al documento como el artefacto universal para el registro del conocimiento, en cualquiera de sus dimensiones y que este tendrá que ser estudiado a medida que evolucione.

Por otra parte, Otlet da una definición más general del libro y del documento, afirmando que son “...un soporte de una cierta materia y dimensión, eventualmente de un cierto plegado o enrollamiento en el que se incluyen signos representativos de ciertos datos intelectuales [...] hay desde ahora un término más genérico (biblión o bibliograma o documento) que cubre a la vez todos los tipos: volúmenes, folletos, revistas, artículos, cartas, diagramas, fotografía, estampas, certificados, estadísticas, incluso discos fotográficos y películas cinematografiadas" (Otlet, 2004, p.43).

El mismo autor ofrece un sentido amplio del documento y también un sentido universal del soporte "las cosas materiales en sí (objetos) pueden ser consideradas documentos cuando se erigen en elementos de estudio o prueba en una demostración. Se trata de entonces de «documentación objetiva» y de «documentación automática»" (Otlet, 2004, p. 217).

Desde esta nueva representación, el Profesor Radamés Linarés afirma que Otlet:

Asentó un concepto más amplio y universal, el documento, y lo concibe de un modo nuevo, como algo que transmite información, principalmente escritos de todo tipo, pero también "cosas" que dependen de los usos a los que conceptualmente fueron destinados. (Linares, 2004, pp. 3-4).

Sobre ese nuevo concepto del documento que postuló Otlet se deja ver muy claramente su valor probatorio de los hechos, sobre este enunciado Buckand determina que para Otlet los documentos son:

Registros gráficos y escritos de los objetos mismos, también pueden ser considerados como "documentos" si llegamos tomar información de ellos observándolo-os. Por ejemplo, objetos tales como artefactos, hallazgos arqueológicos, modelos de juegos educativos y obras de arte. (Buckland, 1997, p. 807, traducido por el autor).

Sobre el compendio de este marco de acotaciones, se puede apreciar que Otlet aclara sobre su objeto de estudio y deja ver muy claramente que la realidad de los hechos, la objetividad y subjetividad del o de los individuos y la necesidad de transmitir el conocimiento, son los tres elementos fundamentales que dan origen a las cosas informativas de creación humana, para la vida. Que la idea ilimitada de que el libro y el documento administrativo eran los únicos portadores de la verdad no era la única panacea para la sociedad, que no significaba que todo el conocimiento humano se encuentre registrado en ellos y que existen otros instrumentos eficaces que albergaban el conocimiento humano. 


\section{La noción de Documentación en el Tratado de Paul Otlet}

Paul Otlet desde finales del siglo XIX es crítico de la bibliotecología, cuestionaba la inflexibilidad de su objeto de estudio, lo cerrado de esta ciencia y sus prácticas informativas, por lo que declara la necesidad de establecer una nueva ciencia que pudiera reconocer y tratar a todos los documentos que registran, intercambian y consumen información para el conocimiento de los individuos, ya que durante ese período crucial de la historia se generaron grandes diferencias en la características de las masas documentales originadas por los procesos industriales, científicos y técnicos. Además de los servicios de información que surgieron en las instituciones informacionales para el acceso al conocimiento científico que:

A estas alturas reclamaba, no sólo conocimientos propios de los procesos bibliográficos, archivísticos y bibliotecarios, y de técnicas y tecnologías avanzadas, sino que exigía también el dominio del tema a tratar, el conocimiento de las regularidades de la información y la comunicación científica y de las técnicas de investigación que facilitan el análisis y la síntesis.(Satién, 1999, p. 68).

La Documentación como ciencia moderna se caracteriza por su sistematicidad, su poder de predicción, y sobre todo por su utilidad práctica que responde a su vez, a la productividad y eficacia informacional y comunicacional sobre los conocimientos registrados por la humanidad. "... Otlet reflexiona [...] e inventa la Documentación como teoría y como práctica trata de integrar y potenciar saberes y actividades ya existentes en torno a una concepción universal de documento para satisfacer necesidades informativas del modo más rápido y eficaz" (López, 2001, p.263).

En 1981 el Profesor José López Yepes desde el concepto otletiano, concibe a la Documentación como:

Una Ciencia para la Ciencia, en cuanto coadyuvadora a establecer las causa últimas de otras ciencias por medio de diferentes instrumentos ofrecidos aquéllas. Todo ello sin perjuicio de que por sí sola procure desarrollar los métodos «ad hoc» de trabajo y los estudios pertinentes para su desarrollo como ciencia. En este sentido, en los postulados y el ambiente general de trabajo de la llamada Ciencia de la Ciencia, la Documentación busca las causas últimas de la comunicación de la ciencia en todos los procesos que posibiliten la transmisión ultima de los conocimientos científicos con el fin de convertirse en fuentes de nuevos conocimientos. Ello sitúa a la disciplina, de un lado, en el ámbito espectral de la Ciencia de la Ciencia, y, de otro lado, dentro de los linderos de las ciencias informativas. (López, 1981, p.702).

Este rasgo de la Documentación desplegada por Otlet se ratifica nuevamente en 1988 por Emilia Currás, donde ella manifiesta que las Ciencias de la Documentación son "experimentales, aplicadas, dinámicas, sociales, humanísticas, históricas y deterministas" y en ellas siempre hay que tener en cuenta sus dos aspectos, teórico y práctico, es decir, científico y técnico; adquieren una dimensión universal con una componente de "interdisciplinariedad, intradisciplinariedad y transdisciplinariedad" afirmando que "la dualidad de soberana y esclava, siempre imprescindible, es la que puede elevar a las ciencias de la documentación a una categoría superior" (Curras, 1988, pp. 109-111 citado en Ros, 2000)

Otra contribución para reiterar la visión otletiana la afirma María Dolores Ayusa en 1995, al afirmar que esta ciencia:

Desarrollaría otras ramas: Biblioteconomía, bibliografía, bibliotécnica, sobre los conceptos fundamentales de la Meta-documentación o Documentación Pura, conceptos que descansan en cuatro elementos: la representación del mundo (de la Documentación), un sistema de signos, la creación de "soportes" prácticos y manejables y finalmente anotaciones que puedan conservarse, comunicarse y difundirse. (Ayuso, 2011, p. 715). 
De acuerdo con las definiciones hasta aquí suscritas y para tener un mejor ámbito de compresión sobre la Documentación como conocimiento científico describiremos algunas ideas, métodos, técnicas e instrumentos propuestos por Otlet para su construcción cientifica.

Comencemos por evocar que Otlet al colocar en el escenario intelectual de su época una postulación que se distancia de la bibliotecología y la archivología, pero que a su vez tomaba elementos de estas y de otras ciencias para llevarla a un plano transdisciplinar, le era imperioso precisar desde el objeto de la lógica y en base a la teoría general y la teoría descriptiva que: "La documentación debe constituirse en cuerpo sistemático de conocimientos como ciencia y doctrina de una parte; de otra en técnica y una tercera parte en cuerpo sistémico de organización" (Otlet, 2004, p. 11). A través de este enunciado Otlet da una mayor compresión de la ciencia que él propone, los métodos y técnicas documentales para llevarlas a una mayor flexibilidad en correspondencia con los artefactos informativos provistos como documentos.

Desde esta perspectiva, Otlet da un rasgo distintivo a la Documentación, su objeto de estudio, el documento en toda su extensión, debido a que su posicionamiento científico se enmarca por ser una: "ciencia general, auxiliar de todas las otras y que les impone sus normas en la que en ellas tiene en fluir resultados en forma de documento" (Ibídem).

En el Tratado de Documentación, Otlet hace una representación muy explícita desde la base radical sobre el término: "Documentación (sustantivo). Acción de documentar o conjunto de documentos" (Ob. Cit. p.13).

Por ello, Otlet planteó el método científico de esta ciencia en dos fases; la directa y la indirectamente, la caracterizó por su posibilidad de contrastación y replicación. Así como la coincidencia del conjunto de datos en los objetos y la acción de generar artefactos para una nueva representación simbólica de estos. Esta apreciación, se distingue en la siguiente declaración formulada por Otlet:

[...] en enumerar los diversos factores que intervienen en el problema propuesto y en dilucidar sucesivamente la influencia de cada uno de ellos tomados aisladamente, siendo todos los demás ahora invariables" (Ob. Cit. p.22).

De allí, enunció la viabilidad de acumulación de recursos informativos, por lo que tiene un estrecho compromiso con los intereses económicos y de los poderes prevalecientes en la sociedad. Esta argumentación se basa en el siguiente teorema enunciados por Otlet: "Todo lo que por convenio y por cooperación pueda llevar a la mayor amplitud y unidad, por tanto a facilitar el trabajo intelectual y el desarrollo del pensamiento..." (Ob. Cit. p.11).

Para el logro de ese designio acumulativo de documentar a los individuos, Otlet fija su forma instrumental, manifestando que "La documentación es una ciencia de observación que, una vez llegada a la expresión de ciertas relaciones generales, se sirve del método deductivo para generalizar sus datos y de los métodos de combinación y de invención para imaginar datos nuevos" (Ob. Cit. p.23).

Frohmann (2008) hace análogo explicito sobre este postulado, exteriorizando que:

Así como los científicos naturales y sociales se deben disciplinar a través de las técnicas del método científico de los hechos que claramente son revelados por medio de la observación rigurosa y la experimentación práctica, los documentalistas se deben disciplinar a través de las técnicas de documentación de la cual la misma forma de hechos se revela con claridad y distinción por medios rigurosamente construidos y un sistema de signos organizado. (p. 78, traducido por el autor)

Por otra parte, Otelt consiente que el discurso de los individuos tiene que ver con las pretensiones del conocimiento y la evaluación de los resultados en relación a la verdad y su adecuación con los hechos, abordó los problemas prácticos de esa moderna ciencia. Declarando que le tocara enunciar métodos propios para resolverlos, que sus esfuerzos se deben centrar en recuperar las verdades originales de los documentos y encuadrarlas en el marco 
sistémico de las ciencias a través del análisis y la síntesis de los elementos que componen al documento, ya que este es el medio de transmitir datos informativos de una realidad y de un conocimiento. (Otlet, 2004, p. 25)

En relación a esta manifestación, Van Acker sostiene que Otlet dio la apertura a nuevos medios de comunicación, ya que:

Con la noción de documentación, Otlet abandona el libro como medios privilegiados para la desimanación de las ideas y afirmó en su lugar la importancia de la multiplicidad y la posibilidad de combinación de medios textuales y visuales. En lugar de ver a todos los medios existentes como diferentes registros de conocimiento. (Van Acker, 2011, p. 36, traducido por el autor y citado en Moura y Lara, 2012).

Santos (2006), afirma que otra de las grandes aportaciones de Paul Otelt, el principio monográfico:

\begin{abstract}
Para realizarla utiliza dos conceptos el de soporte e información (documento) y contenido informacional (mensaje), permitiendo desde esta manera relacionar información que se encuentra distribuida en diversos soportes proporcionando nuevas direcciones. Obteniéndose, con esos procedimientos, nuevos documentos, que contienen también nueva información. Existe aquí, por tanto, un doble movimiento: desmaterialización y virtualización de documentos y su transformación en información nueva, fruto de recombinación de informaciones, que irá conformando un nuevo documento. (p. 89, traducido por el autor).
\end{abstract}

No cabe duda alguna de que Otlet muestra la utilidad verdadera y objetivamente de los documentos, que estos pueden probar algún hecho y que a ellos se le pueden identificar fácilmente. Es sólo un asunto de normalizar los procesos de análisis y organización sobre el contenido informativo de los documentos.

Sobre este punto en particular Moura y Lara, determinan que:

Para Otlet, esto es, el aislamiento de elementos de información es una cuestión técnica de "institucionalizar los procesos": se trata de aplicar un método desarrollado "científicamente" y previamente dotado de coherencia teórica y metodológica, y organizar las instituciones responsables por su aplicación y las relaciones entre ellas. (Moura y Lara, 2012, p. 6, traducido por el autor)

Otro ángulo útil en el que Otlet también reflexionó y adapto, es la dimensión humana del hombre y su grado de satisfacción, puntualizó que "La documentación debe tender a realizar el máximo de condiciones para el hombre, cuyo límite alcanzar sea la ubicuidad, la eternidad y el conocimiento intuitivo" (Otlet, 2004, p. 25). Desde una ciencia operativa que permitiese realizar cálculos, predicciones y actuación a través de la experimentación y matematización.

También este mismo autor consideró y amoldo para la documentación la teoría basada en las ciencias puras. Al explicar que la meta-documentación o documentación pura permitirá elevar la documentación como ciencia y que no debe ser estática y que se debe transformarse en otras especializaciones o subespecializaciones de forma gradual de acuerdo al contexto en que esta se encuentre, en palabras de Otlet:

Que no hay que detenerse en razonamiento e invención por el único deseo de llegar a resultados eminentemente prácticos y generalmente aplicables, sino de tratar de llegar más lejos. [...] La Documentación pura debe de reivindicar la posibilidad de elevarse tan alto como puedan las facultades, no de todos, sino de algunos, de llegar a transcripciones documentales raras o únicas de las combinaciones de los documentos complicados e inusuales. Mañana, es muy probable, sabrá simplificar, generalizar y sacar lo útil de lo que hoy sería verdadero y racional. (Otlet, 2004, p. 26). 
Con respecto a las ciencias sociales, Rayward afirma que:

Un aspecto de la visión de Otlet es la forma en que la organización y difusión de conocimiento puede contribuir a un mundo pacífico, unificado. Porque, densamente organizada y escrita la idiosincrásica que sea, es quizás una de las primeras introducciones generales al estudio de la información como un fenómeno social importante. (Rayward, 1991,p.138, traducido por el autor).

Frohmann (2011) hace un punto similar:

Otlet vio los documentos en todas partes, no sólo en los fondos de las bibliotecas y los productos de las editoriales, también en la fotografía, microformas, el cine, la radio, la televisión e incluso los rayos $\mathrm{x}$. Estaba convencido de que la documentación - nombre que le dio a un nuevo profesional, práctica bibliográfica y sus instituciones asociadas - es una fuerza profundamente social. (p. 75, traducido por el autor).

Dentro de estos marcos teóricos, Otlet (2004) también se apoya y ajusta la teoría de la evolución humana la discernir que "Sin el lenguaje, la inteligencia no habría podido perfeccionarse, sin la escritura-documentación el lenguaje habría quedado en un estado inferior" (p. 27). Expresando en este sentido que el lenguaje, el pensamiento científico, los soportes y los medios de registrar el conocimiento evolucionaron a través del tiempo por las innovaciones morfológicas y el pensar simbólico.

Otlet igualmente se orientó y se adecuo sobre la concepción interdisciplinaria, construyó un marco de referencia conceptual en el que se basó en principios e investigaciones empíricas de nueve disciplinas: Bibliotecología, Archivología, Museología, Lingüística, Sociología, Lógica, Psicología, Tecnología y Pedagogía. Además creía necesario considerar otras ciencias que sean ineludibles para poder estudiar y comprender la conexión física y funcional del documento y la documentación.

Habría que decir también que Van den Heuvel (2011) discierne que Otlet considero y utilizó a la arquitectura mediante la obtención de símbolos, los cuales tuvieron grandes cambios a raíz de las revoluciones políticas e industriales que acontecieron en Europa durante el siglo XVIII hasta principios del XX, donde surgen nuevos sistemas de símbolos los cuales transmiten nuevo significados acorde a los cambios sociales (p. 129, traducida por el autor).

Esa alusión la concibe este autor porque Otlet describió las teoría de arquitectura por el carácter cambiante de los soportes y los signos de registros, esta se pueden apreciar en el postulado que se encuentra en Tratado de Documentación de la siguiente manera: "Las características del libro debe ser una "estructura de ideas", de los datos intelectuales, lleva a tomar consideración la enorme revolución realizada en nuestros días por la misma arquitectura" (Otlet, 2004, p. 100).

Cabe señalar que Otlet (2004) considero nuevamente la teoría de la evolución y además los avances tecnológicos en relación a la producción en masa, en las cuales se basa para dejar plasmada unas de las ideas futuristas en su Tratado de Documentación, al referirse sobre las máquinas o sobre el cerebro mecánico y su manera de comunicación al afirmar:

Debemos contar un complejo de máquinas asociadas: $1^{a}$ La transformar el sonido en escritura. $2^{a}$ La multiplicación de dicha escritura tantas veces a haga falta. $3^{a}$ La organización de los de documentos de forma que cada dato esté individualizado, se encuentre en relación con los restantes datos del conjunto y se pueda recuperar cuando sea necesario. $4^{\mathrm{a}}$ El índice de la clasificación unido a cada dato; perforación del documento en función con estos índices. $5^{a}$ Clasificación automática de los documentos y colocación de los mismo archivadores $6^{\text {a }}$ Recuperación automática de los documentos a presentar y presentación de los mismos, a la vista 
o en la parte de una máquina que le añada nuevas anotaciones. $7^{\text {a }}$ Manipulación mecánica de todos los datos registrados para tener nuevas combinaciones de hechos, nuevas relaciones de ideas y nuevas operaciones con la ayuda de los números. (p. 391).

Sobre esta formulación es necesario contemplar el esbozo realizado por Salvador y Arquero (2006) sobre la recuperación de la información a partir del punto de vista otletiano:

Esta hipótesis presenta la visión futurista de Otlet sobre el acceso automático a distancia a la documentación existente en el mundo con base en los siguientes elementos: una pantalla, un teléfono, un inmenso edifico donde se encuentran los libros e información, y personal altamente calificado para seleccionar y combinar la información. Evidentemente, lo que Otlet profetizó era lo que hoy denominamos teledocumentación o recuperación de la información en línea, en la que desde un ordenador y a través de redes de telecomunicaciones, un profesional, o bien el propio usuario, accede a la información contenida en bases y bancos de datos (tratados a su vez por personal especializado) y que aparecen al instante en el ordenador. (p. 36).

Otlet (2004) también acudió y ajusto a la teoría psicodinámicas para la denominación ese nuevo profesional al referirse que:

Dentro de las diversas ramas de la documentación, en sus diferentes etapas y momentos, existe un personal numeroso y excesivamente especializado, [...] se necesitaba de un término genérico para que abarque a todas las personas relacionadas con los libros y la documentación. Se ha probado con el neologismo "documentalista" (p. 393).

En síntesis, los aspectos tratados en este artículo dejan ver el génesis de una ciencia incuestionable guiada por procesos intelectuales y basada en métodos universales y expandibles con disposición prescriptiva y descriptiva para su objeto de estudio, el documento como cosa operativa en base a la información registrada y que se sirve de la ciencias naturales y de la ciencias sociales porque su utilidad está aprehendida a la realidad social en toda su complejidad y dinamismo donde la estabilidad de los métodos será el resultado de atravesar una serie de medios coherentes que desarrollen y fortalezcan la documentación de una manera más integrada.

\section{Conclusión}

El Tratado de la Documentación se publica en 1934 en un escenario marcado, por una parte, una fuerte revolución tecnológica que modificó los soportes tradicionales de registro de información, y por otra, por la influencia de los enfoques positivistas en la Bibliotecología y Archivología.

En El Tratado de la Documentación Paul Marie Ghislain Otlet expone una metodología de cómo hacer que el conocimiento registrado estuviera disponible para quien lo necesitara y con ello contribuir al enriquecimiento intelectual de la Humanidad. En tal sentido Otlet buscó constituir, difundir y consolidar un saber científico que fuera más allá de los presupuestos tradicionales de la Bibliotecología y la Archivología, que se integran en el campo de conocimientos denominado por él Documentación. 


\section{Bibliografía}

Ayuso, M. D. (1995). Conceptos fundamentales de la Teoría de la Documentación. Estudio Terminológico y Versión Española del Traité de Documentation de Paul Otlet. Disertación (Doctorado). Faculta de Letras. Universidad de Murcia.

Buckland, M. K. (1997). What Is a “Document”? JASIS, Vol. 48. No.9.: 804-809.

Cabrales, G. \& Linares, R. (2005 Ene - Ago.) Origen y evolución de la Ciencia de la Información (1895-1962). Biblios. Año 6. (2122): 84-98. Recuperado el 22 de diciembre de: http://redalyc.uaemex.mx/pdf/161/16102207.pdf

Currás E. (1998). La información en sus nuevos aspectos. Madrid: Parainfo.

Espely, R. (2011). 'The times are wrong': Paul Otlet, modernist anachronism or prophetic knowledge architect of the postmodern? (Tesis de maestría, Universidad de Gales) Recuperado el 27 de diciembre de 2012, de http://cadair.aber.ac.uk/dspace/handle/2160/7731

Frohmann, B. (2011) The Role of Facts in Paul Otlet's Modernist Project of Documentation. Recuperado el 03 de febrero 2013 de: http://faculty.fims.uwo.ca/frohmann/BFhome/Documents/Role\%20of\%20Facts proof.pdf

(2004). Bibliotecología y Ciencia de la Información: ¿subordinación, exclusión o inclusión? ACIMED. Vol. 12. No. 3. Recuperado el 3 de febrero de 2013 de http://bvs.sld.cu/revistas/aci/vol12 3 04/aci07304.htm

López, J. L. (1981). ¿Que es documentación?. Anabad. Vol. 31. No. 4. 701-707. Recuperado el 02 de febrero de 2013 de http://dialnet.unirioja.es/descarga/articulo/965324.pdf

(1997 ene. - jun.). Reflexiones sobre el concepto de documento ante la revolución de la información: ¿un nuevo profesional del documento?. Scire. Vol. 3. No. 1.: 11-29. Recuperado el 02 de febrero de 2013 de http://www.ibersid.eu/ojs/index.php/scire/article/view/1064/1046

(2001). La investigación española en Teoría de la Documentación (1990 2000). Revista General de Información y Documentación. > Vol. 11, No 2.: 259-280. Recuperado el 05 de febrero de 2013 de http://revistas.ucm.es/index.php/RGID/article/view/RGID0101220259A.

Malheiro, A. (2006). Informação. Da compreensão do fenómeno e construção do objecto científico. Porto: Afrontamento.

Moura, A. P. \& Lara, M. L. G. d. (2012). Construir o edifício documentário: concepções de Paul Otlet para uma ciência e uma técnica dos documentos. Perspectivas em Ciência da Informação, 17(4), 2-17. Recuperado el 08 de febrero de 2013 de: http://portaldeperiodicos.eci.ufmg.br/index.php/pci/article/view/1531

Otlet, P. M. (2004). El Tratado de Documentación: el libro sobre el libro: teoría y práctica, traducción de María Dolores Ayuso García. La Habana: Félix Valera. (Trabajo original publicado en 1934).

Rayward, W. B. (1975). The universe of information: the work of Paul Otlet for documentation and international organisation. Moscow: All-Union Institute for Scientific and Technical Information (VINITI), for International Federation for Documentation (FID).

(1991). The Case of Paul Otlet, Pioneer of Information Science, Internationalist, Visionary: Reflections on Biography. Recuperado el 28 de diciembre de 2012 de http://people.lis.illinois.edu/ wrayward/otlet/PAUL OTLET REFLECTIONS ON BIOG.HTM

(1997) The Origins of Information Science and the International Institute of Bibliography/International Federation for Information and Documentation (FID). Journal of the American Society for Information Vol. 48 № 4 (April 1997): 289-300. Recuperado de http://people.lis.illinois.edu/ wrayward/OriginsofInfoScience.pdf

Rodríguez, B. (2002). Revisión de las clasificaciones documentales basadas en el soporte. Revista Española de Documentación Científica. Vol. 25. No. 1.: 74-86. Recuperado el 02 de febrero de 2013 de http://redc.revistas.csic.es/index.php/redc/article/view/87/149

Ros, J. (2000). El concepto de Documentación en la doctrina española. Primer Congreso Universitario de Ciencias de la Documentación: Teoría, historia y metodología de las ciencias de la documentación.: 317-349. Madrid, España. Recuperado de http://www.ucm.es/info/multidoc/multidoc/revista/num10/paginas/pdfs/Jros.pdf

Salvador, J. A., \& Arquero, R. (2006). Una aproximación al concepto de recuperación de información en el marco de la ciencia de la documentación. Investigación Bibliotecológica, Vol. 20. No. 41.: 13-43. Recuperado el 06 de febrero de 2013 de http://www.ejournal.unam.mx/ibi/vol20-41/IBI002004101.pdf 
Santos, P. d. M. (2006). Dos. O ponto de inflexão Otlet: uma visão sobre as origens da Documentação eo processo de construção do Princípio Monográfico. 2006. 138 f. Disertación (Maestría en Ciencia de la Información). Escuela de Comunicación y Artes, Universidade de San Paulo.

Satién, E. (1999 ene. - jun). Reflexión sobre la gestión de innovación bibliotecológica: raíces, esencias, cambios. Scire. Vol. 5 No.1. Recuperado el 31 de enero de 2013 de: www.ibersid.eu/ojs/index.php/scire/article/download/1107/1089

Van den Heuvel, C. (2011) Building Society, Constructing Knowledge, Weaving the Web: Otlet's Visualizations of a Global Information Society and His Concept of a Universal Civilization. Recuperado el 04 de Febrero de:

http://citeseerx.ist.psu.edu/viewdoc/download?doi=10.1.1.177.7553\&rep=rep1\&type=pdf

\section{Datos del autor}

\section{Leomar José Montilla Peña}

Encargado de la Biblioteca Especializada del Instituto Nacional de Investigaciones Agrícola, Unidad Ejecutora Lara, Venezuela. Profesor Instructor del Programa de Formación en Ciencias de la Información de la Universidad Politécnica Territorial Andrés Eloy Blanco, Barquisimeto, Venezuela. Integrante del Comité Interinstitucional para el Fortalecimiento de la Gestión del Programa Nacional de Formación en Ciencias de la Información, Ministerio del Poder Popular para la Educación Universitaria, Caracas, Venezuela.

leomonp@gmail.com

$\begin{array}{ll}\text { Recebido-Received } & : 2013-05-15 \\ \text { Aceito-Accepted } & : 2013-06-30\end{array}$

New articles in this journal are licensed under a Creative Commons Attribution 3.0 United States License.

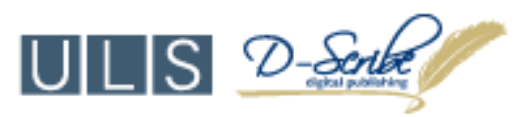

This journal is published by the University Library System of the University of Pittsburgh as part of its D-Scribe Digital Publishing Program and is cosponsored by the University of Pittsburgh Press. 